\title{
Genioglossus and Diaphragm Activity during Obstructive Apnea and Airway Occlusion in Infants
}

\author{
ESTELLE B. GAUDA, MARTHA J. MILLER, WALDEMAR A. CARLO, JULIANN M. DiFIORE, AND \\ RICHARD J. MARTIN \\ Department of Pediatrics at Rainbow Babies and Childrens Hospital. Case Western Reserve University, \\ Cleveland, Ohio 44106
}

\begin{abstract}
To document mechanisms contributing to upper airway collapse, we compared the electromyographic activity of the genioglossus (GG) and diaphragm (DIA) during spontaneous mixed and obstructive apnea and during induced end-expiratory airway occlusion in 11 premature infants. In addition to ventilation and esophageal pressure measurements, we obtained DIA and GG electromyograms (EMG) from subcostal and sublingual surface electrodes, respectively. Amplitude of the DIA EMG and the frequency of occurrence of the GG EMG were determined for: $I$ ) the breath preceding apnea or occlusion, 2) the initial and last obstructed inspiratory efforts, and 3) the first breath at resolution of both apnea and occlusion. During spontaneous apnea with airway obstruction amplitude of the DIA, EMG decreased on the initial obstructed inspiratory effort and did not exceed that of the breath preceding apnea until reestablishment of flow. In contrast, during end-expiratory airway occlusion, the amplitude of the DIA EMG increased both during and at release of occlusion. In $18 \pm 6 \%$ of the spontaneous apneic episodes, GG EMG was present with the breath preceding apnea and this frequency did not increase significantly until resolution of the apnea. During induced airway occlusion, GG EMG was not present with the breath preceding occlusion but its frequency did increase to $58 \pm 8$ and $42 \pm 8 \%$ with the last occluded inspiratory effort and the first breath after release of occlusion, respectively. The decreased presence of the GG EMG from the last occluded effort to the breath at release of occlusion ( $58 \pm 8$ versus $42 \pm 8 \%, p<$ $0.05)$ was probably due to the greater mechanoreceptormediated inhibition associated with reestablishment of flow. This study thus demonstrates markedly different respiratory muscle responses to spontaneously occurring obstructed inspiratory efforts during apnea as compared to experimentally induced airway occlusion in preterm infants. (Pediatr Res 26: 583-587, 1989)
\end{abstract}

Abbreviations

DIA, diaphragm

GG, genioglossus

EMG, electromyogram

Premature infants are known to exhibit pharyngeal obstruction during spontaneous apnea (1). It has been postulated that upper

Received February 8, 1989; accepted July 21, 1989.

Cortespondence Richard J. Martin, M.B., F.R.A.C.P., Department of Pediatrics

Rainbow Babies \& Childrens Hospital, 2101 Adelbert Road, Cleveland, Ohio 44106 Supported by N1H Grant HL25830. airway obstruction occurs when activity of upper airway dilating muscles is absent or decreased and activity of chest wall muscles is normal or increased $(2,3)$. We have recently reported, however, that phasic activity of the GG (a pharyngeal dilating muscle) is usually absent during unobstructed breathing in premature infants (4). Even when $G G$ activity was evoked by end-expiratory airway occlusion, premature infants with mixed and obstructive apnea had less activation of their GG than control infants (4). A reduction in central respiratory drive to the muscles of respiration in the apneic infants may have accounted for this difference because premature infants with apnea have been shown to have a decreased slope of their $\mathrm{CO}_{2}$ response curve (5). Upper airway muscle and DIA activity have not, however, been directly measured during spontaneous apnea associated with airway obstruction in premature infants.

The neuromuscular activity of the upper airway muscles and DIA is influenced by the mechanoreceptor-mediated inhibition that occurs during lung inflation $(6-10)$. Thus, the technique of end-expiratory airway occlusion has been used to determine the strength of this inhibition on respiratory muscle activity $(4,6,9$ 12). Using this technique in premature infants, we have previously documented activation of the GG and a prolongation of inspiratory time during induced occlusion, suggesting that mechanoreceptor-mediated inhibition of lung inflation alters the activity of upper airway and chest wall muscles $(4,6)$. In our study, we performed end-expiratory airway occlusions to document the response of the DIA and GG to airway obstruction during periods of spontaneous ventilation. In the same infant population, we recorded the electromyographic activity of the DIA and GG during spontaneous mixed and obstructive apneic episodes.

The aim of our study was to assess the relative changes in upper airway and DIA activity before, during, and at resolution. of a spontaneous apnea with airway obstruction and to compare these findings to upper airway and DIA activity in response to experimentally induced nasal occlusion. Our data indicate that in premature infants the amplitude of DIA activity is decreased during spontaneous mixed and obstructive apnea; whereas the frequency of occurrence of GG activity is only increased at resolution of apnea. However, in response to experimentally induced occlusion, DIA and GG activity are both increased during occlusion and remain elevated at release of the occlusion.

\section{MATERIALS AND METHODS}

The study population consisted of 11 infants with a history of three or more bradycardic episodes (with or without documented apnea), as detected by ECG and impedance monitoring during the $24 \mathrm{~h}$ before the study period. Monitor alarms were set for heart rate less than 80 beats/min and apnea for more than $20 \mathrm{~s}$, 
with bradycardia assumed to represent obstructive apnea. The infants had a mean $( \pm S D)$ gestational age of $28 \pm 2 \mathrm{wk}$ (range 26-31 wk), a birth wt of $1200 \pm 300 \mathrm{~g}$ (range 780-1700 g), and were studied at a postconceptional age of $32 \pm 1$ wk (range $31-$ $34 \mathrm{wk}$ ). None of the infants required supplemental oxygen, and none exhibited tachypnea or radiographic evidence of lung disease at the time of the study; nine of the 11 infants were receiving theophylline for the treatment of apnea of prematurity with a mean $( \pm S D$ ) theophylline level of $6.5 \pm 1 \mu \mathrm{g} / \mathrm{mL}$. The infants were studied in the Neonatal Pulmonary Research Laboratory after informed consent was obtained from the parents. Measurements were performed during sleep without the use of sedation with the infant in an incubator that provided a neutral thermal environment. Behavioral criteria were used to assess sleep state with active sleep associated with rapid eye movements, body movements, and an irregular respiratory pattern. Quiet sleep was characterized by a regular respiratory pattern and the absence of rapid eye and body movements with the exception of occasional startles (13). Written and informed parental consent was obtained from one or both parents on a standard form approved by the Institutional Research Review Board.

GG and DIA EMG were measured noninvasively with surface electrodes. The apparatus used to record the GG EMG consisted of two silver, domed, 4-mm surface electrodes attached to flexible insulated wires and mounted on a moulded acrylic chin piece that was held in place by an elastic head band (4). The wires could be adjusted to allow a secure fit for each infant. The two surface electrodes were placed in an anterior sublingual position on each side of the frenulum. The infants tolerated the apparatus well and were positioned with the head turned laterally and the neck in a neutral position. The DIA EMG was obtained from two adhesive surface electrodes (Medtronic Andover Medical, Lowell, MA) placed $5 \mathrm{~mm}$ apart on or slightly below the right costal margin between the mid- and anterior axillary line such that the best inspiratory EMG with an optimal signal-to-noise ratio was obtained. The GG and DIA EMG were amplified (Preamplifier, Coulbourn, Lehigh Valley, PA) and displayed on an oscilloscope. Raw EMG were filtered from $30-300 \mathrm{~Hz}$. The electrocardiographic artifact was removed by gating (SG-1 EKG Blanker, CWE, Inc., Ardmore, PA) from the DIA EMG (4). Both EMG were averaged by a moving time averager (14) (MA821 Moving Averager, Charles Ward Enterprises, PA) with a time constant of $100 \mathrm{~ms}$. Esophageal pressure was measured by a dome transducer (Gould) attached to a 5 Fr fluid-filled catheter (Argyle, St Louis, MO) placed in the midesophagus. Airflow and mask pressure were measured with a nasal pneumotachograph with a linear pressure-flow relationship up to $5 \mathrm{~L} / \mathrm{min}$, a resistance of $0.78 \mathrm{kPa} \mathrm{I}^{-} \mathrm{s}$, and a dead space of less than $2.5 \mathrm{~mL}$ (15). The averaged GG and DIA EMG, esophageal pressure, airflow, and mask pressure were recorded on a 6-channel Gould chart recorder.

Spontaneously occurring apneic episodes were defined as obstructive if there was absence of nasal airflow with persistent DIA EMG activity and esophageal pressure swings and mixed if there was a combination of obstructive inspiratory efforts and episodes of absent nasal airflow, esophageal pressure swings, and diaphragmatic activity of at least $2 \mathrm{~s}$ duration. All spontaneous apneic episodes occurred during active sleep and had their onset at a time when oxygen saturation exceeded $90 \%$. Experimentally induced airway occlusion during sleep was performed on each infant by manually occluding the inflow and outflow port of the nasal mask at end-expiration for $10 \mathrm{~s}$. Experimental occlusions were separated by at least 60-s intervals and were only performed when oxygen saturation was $95 \%$ or more as measured by pulse oximetry (Nellcor-100, Nellcor Incorporated Haywood, CA). Occlusions and apnea were always separated by at least $60 \mathrm{~s}$. A mean $( \pm S D)$ of $5 \pm 3$ mixed and obstructive apneic episodes (range 2-10) of $14 \pm 5 \mathrm{~s}$ duration (range 7-25s) and $6 \pm 1$ endexpiratory occlusions (range 3-6) of 10 s duration were analyzed per infant. An equal number of experimental occlusions were performed in active and quiet sleep for each infant. Spontaneous apnea was never associated with arousal. Arousal did occasionally occur during occlusion, but the data were not subjected to further analysis.

During apneic episodes with spontaneous airway obstruction and experimentally induced airway occlusions, we measured the amplitude of the DIA EMG and how often the GG EMG was present for $/$ ) the breath preceding the spontaneous apnea or experimental occlusion, 2) the initial and last obstructed inspiratory efforts, and 3 ) the first breath after resolution of the apnea or release of occlusion. The DIA amplitude of all breaths was expressed as a percent of the amplitude of the breath preceding the apnea or occlusion which was designated as $100 \%$. The GG EMG was analyzed differently than the DIA EMG because a phasic GG EMG was frequently absent; a GG EMG preceded less than $5 \%$ of the experimental occlusions and less than $20 \%$ of the spontaneous apneic episodes. Therefore, we analyzed the frequency of occurrence with which a $\mathrm{GG}$ EMG was present (expressed as a percent) in association with each breath or obstructed inspiratory effort, rather than the amplitude of the GG EMG.

Experimentally inducing airway occlusion at end-expiration allowed us to document mechanoreceptor-mediated influences on the activity of the DIA. Inspiratory time was measured from the onset to the peak of the esophageal pressure swing. The inspiratory time of the preocclusion breath was compared to that of the initial occluded inspiratory effort, and the inspiratory time of the last occluded effort was compared to that of the breath at release of the occlusion. Comparative measurement of inspiratory time was also performed for breaths or inspiratory efforts associated with spontaneous apnea.

In our previous data and those of Cohen and HendersonSmart (16) no differences in the GG response to occlusion were observed between active and quiet sleep (4). In our study the DIA and GG responses to occlusion were also comparable between active and quiet sleep, and therefore the occlusion data were combined between states. A mean response during both spontaneous apnea and induced occlusion was calculated separately for each infant and the combined means were then subjected to statistical analysis. Each EMG was analyzed separately during apnea and occlusion by one-way analysis of variance with repeated measures using the Newman-Keuls procedure as appropriate. Comparison of each EMG during apnea versus occlusion was via two-way analysis of variance, with repeated measures. Inspiratory time data were analyzed by Student's paired $t$ test. Statistical significance was defined as $p<0.05$. Results are presented as mean $\pm \mathrm{SEM}$.

\section{RESULTS}

During spontaneous apnea, the DIA amplitude of the initial obstructed effort was only $80 \pm 3 \%$ of the DIA amplitude of the breath preceding the apnea $(p<0.05)$ (Figs. 1 and 2 ). In addition, the DIA amplitude of the last obstructed effort $(96 \pm 8 \%)$ did not differ from that of the breath preceding the apnea. At resolution of the apnea, however, the DIA amplitude of the breath associated with reestablishment of flow was $135 \pm 9 \%$ of the DIA amplitude of the breath preceding the apnea $(p<0.01)$ and was also more than the DIA amplitude of the last obstructed effort ( $135 \pm 9 \%$ versus $96 \pm 8 \% p<0.01$ ) (Fig. 2). Thus, the amplitude of the diaphragmatic EMG decreased at the onset of the apnea and did not exceed that of the preapnea breath until resolution of the apnea.

During experimentally induced airway occlusion, the DIA amplitude of the initial occluded effort $(105 \pm 5 \%)$ did not differ from that of the DIA amplitude of the breath preceding the occlusion (Figs. 2 and 3). However, the DIA amplitude of both the last occluded effort $(145 \pm 13 \%, p<0.01)$ and the breath at release of the occlusion $(130 \pm 12 \%, p<0.05)$ was significantly 